\title{
Entrevista clínica en Pediatría: teoría y práctica
}

\author{
ML. Arroba Basanta ${ }^{a}$, R. Dago ${ }^{b}$, P. Manzarbeitia ${ }^{c}$ \\ aCS Pozuelo Estación. Pozuelo de Alarcón, Madrid. Universidad Autónoma de Madrid. España. \\ ${ }^{b}$ CS Castilla La Nueva. Fuenlabrada, Madrid. España. \\ ¿Universidad Complutense de Madrid. España.
}

\begin{abstract}
Resumen
En la época de formación, durante la carrera de Medicina, los aspectos relativos a la comunicación humana han caído sistemáticamente en el olvido: se asume que un profesional de la salud sabe entrevistar. Esta visión, sin duda idealizada, conlleva otra fantasía: pensar que sea cual sea el tipo de pregunta o la forma de dialogar con el niño y las familias se obtienen resultados similares. La entrevista clínica $(E C)$ es considerada por algunos como un medio para obtener datos significativos, negando las emociones y los sentimientos que encierra toda relación humana. Sin negar el papel instrumental de la EC, entrevistamos, en efecto, para conseguir algo, aunque este "algo" sea a veces simplemente una comunicación. La entrevista en sí misma debe ser objeto de investigación científica y solo entonces seremos capaces de aplicar estrategias de aprendizaje capaces de mejorar nuestro perfil.
\end{abstract}

Palabras clave: Entrevista clínica. Habilidades de comunicación. Escucha activa.

\section{Clinical interview in pediatrics: theory and practice}

Abstract

Human communication skills have been systematically forgotten during medical training at University. It is assumed that a professional involved in health knows how to conduct an interview. This view, undoubtedly idealized, takes along another fantasy: to think that whatever the question is or the way to dialogue with a kid or his/her family, the results are similar. Clinical interview (CI) is considered by some as a way to obtain significant data, without taking into account any emotions and feelings that any human relation has. In fact, we interview, unregarding the instrumental role of $\mathrm{Cl}$, to obtain something, even though this "something" is sometimes merely a communication. The interview itself must be the object of scientific research, only then we will be able to apply learning strategies useful to improve our skills.

Key words: Clinical interview. Resources in communication. Active listening.

María Luisa Arroba Basanta: mlarroba@pap.es

Las autoras declaran no presentar conflictos de intereses en relación con la preparación y publicación de este artículo. 


\section{Introducción}

Al médico especialista en Pediatría se le suponen algunas características personales: que le "gusten" los niños, que sepa tratarlos y comunicarse bien con ellos, que comprenda a los padres y a los abuelos, y sobrelleve con satisfacción su relación con ellos. En ocasiones, también debe saber relacionarse y compartir información con familias "diferentes", ya sea por su estructura o por su procedencia sociocultural. Cuando terminamos la especialidad, nos enfrentamos a la realidad: muy pocos pediatras han tenido la oportunidad de recibir un aprendizaje reglado en comunicación. Todos tenemos diferentes estilos de entender la relación clínica e intentamos hacerlo lo mejor posible dentro de nuestra realidad asistencial. Pero la escasez de formación en habilidades de comunicación puede favorecer que los pediatras perdamos con el tiempo las ganas de "buen hacer" que teníamos al inicio del ejercicio profesional. Es inevitable que los clínicos encuentren, a lo largo de su vida profesional, problemas de los pacientes y/o sus familias frente a los que no sepan qué hacer. El entrenamiento para afrontar esas situaciones forma parte del aprendizaje de las técnicas de comunicación ${ }^{1-3}$.

Se asume que un pediatra sabe entrevistar. Esta visión idealizada conlleva otra fantasía: pensar que sea cual sea el tipo de pregunta o la forma de dialogar con los niños y las familias se obtienen resultados similares.

Hay cuestiones que todos los pediatras nos hemos planteado alguna vez: ¿podemos mejorar la relación con los niños y sus familias?, ¿sabemos comunicarnos con nuestros pacientes?, ¿qué características tenemos como entrevistadores?, ¿por qué, si hemos establecido un diagnóstico de viriasis, la madre sale de la consulta y dice por el móvil al marido o la abuela: "Me han dicho que no tiene nada"? Creo que a todos nos ha pasado que, al intentar crear un ambiente de confianza con los niños y sus padres, hemos tropezado con situaciones de confusión de límites (¿tuteo con falta de respeto, adjetivos como "chata" o "nena"?). Cuando hemos sido pacientes, ¿sabíamos quién se dirigía a nosotros y su nombre?, ¿cómo nos sentimos?

Muchos tratamientos se administran mal o no se cumplen adecuadamente. Cuando tratamos de instaurar o modificar hábitos, ¿las personas cambian sus comportamientos solo porque les digamos que deben hacerlo?

El conocimiento de las habilidades de comunicación facilita una conducta sistematizada que previene o ayuda a re- 
solver estos conflictos. Una relación clínica de calidad mejora la eficacia en la detección y resolución de problemas clínicos, la satisfacción con la atención recibida, y la adherencia al tratamiento ${ }^{4-6}$.

\section{La competencia profesional}

La comunicación es un proceso interpersonal complejo de importancia primordial en la asistencia sanitaria. Los médicos, en la práctica diaria, mantenemos relación con un gran número de personas (pacientes y sus familias, otros miembros del equipo de trabajo, distintos colegas y otros profesionales de la salud), con las que necesitamos comunicarnos de una manera eficaz. La comunicación profesional tiene lugar en diferentes ámbitos, pero el intercambio más frecuente y característico se produce en la consulta, y es allí donde vamos a situar los conceptos, actitudes y técnicas, que expondremos en las siguientes líneas.

Para realizar una consulta realmente eficaz, hemos de ser capaces de integrar cuatro aspectos que, en conjunto, determinan la competencia profesional ${ }^{1,7-10}$ : los "conocimientos médicos", las habilidades de comunicación, la habilidad para tomar decisiones y el manejo de las técnicas de exploración.

Estos cuatro componentes de la competencia clínica están intrincados entre sí y todos ellos son necesarios para la buena práctica de la Medicina. No es suficiente, por ejemplo, poseer grandes conocimientos médicos, si tenemos dificultades de comunicación que impiden descubrir el motivo de consulta, o que el paciente entienda el plan terapéutico, o que salga de la consulta sin intención de ponerlo e práctica. Todo el saber médico se trasmite cara a cara con los pacientes y aquí es igual de importante lo que se dice que el cómo se dice. Las palabras que elegimos, el tono de voz que empleamos y los gestos con que las acompañamos trasmiten al enfermo tanta o más información que las propias palabras.

\section{Características del entrevistador ${ }^{1,5,10-14}$}

Habitualmente, los pediatras pensamos en la calidad técnica de nuestra tarea (medicina basada en pruebas, protocolos...), y es evidente que esto es importante y constituye una condición sine qua non de nuestro trabajo pero, en general, esta calidad se nos supone y es difícil de ser evaluada por el usuario, a no ser que sea muy deficiente.

Los pacientes valoran fundamentalmente:

- Un médico amable: cordialidad. Es la manera de relacionarse entre personas socialmente educadas y 
que se respetan. Incluye en su definición amabilidad, consideración, respeto y atención. El paciente espera ser tratado como persona, no como un número más de la lista, debe sentirse bienvenido aunque sea "el 45". Las habilidades para demostrar cordialidad están en mirar al paciente, en el tono de voz, en llamarle por su nombre y en estrecharle la mano.

- Un médico humano: empatía. Es la capacidad del profesional para reconocer las emociones del paciente y trasmitirle que le importan.

- Un médico que escuche: reactividad. Es la capacidad del profesional para escuchar de una manera bidireccional. Las habilidades para ponerla en práctica son:

- Dejar que el paciente y sus padres nos expliquen con sus propias palabras el motivo de consulta.

- No interrumpirle a mitad del relato. Esta comprobado en numerosos estudios que no hablan más de dos minutos y transmiten una información muy valiosa para el desarrollo posterior de la consulta. - Facilitar la libre narración mostrando interés con elementos verbales ("hmn, hmn", "cuénteme", “¿y eso?", repetición de palabras clave) y no verbales (mirada atenta, cabeceos). No permitir que escribir en la historia o el uso del ordenador inhiban la libre comunicación.

- Empleo apropiado de los silencios. Es un error creer que los silencios deben evitarse. Cuando el paciente no sabe que decir o se siente inseguro, el médico debe resistir la tentación de interrumpir formulando una nueva pregunta o abordando otras cuestiones, y dejar que sea el paciente quien elabore la información.

- Un médico del que se pueda fiar y sentir que está en buenas manos: asertividad. Es la capacidad de transmitir credibilidad y seguridad al paciente. Las habilidades para ponerla en práctica en la consulta son:

- Tener en cada situación una respuesta emocionalmente tranquila a las quejas, demandas y miedos de nuestros pacientes, porque sabemos como profesionales lo que tenemos que hacer.

- No utilizar respuestas agresivas cuando dudan de nuestra competencia. Si en algún momento el caso supera nuestra capacidad clínica, hay que saber pedir ayu$\mathrm{da}$, sin sentirse culpable por ello. 
- Una última característica cuya necesidad aumenta con la edad y el número de años de ejercicio profesional: paciencia.

\section{Objetivos}

Los objetivos de la EC en la atención pediátrica son ${ }^{1,15-17}$ : conocer el motivo por el que el paciente acude, prescribir un tratamiento o recomendar una pauta de conducta, y establecer una relación asistencial satisfactoria para el pediatra, el paciente y sus familiares.

Existen diferentes tipos de entrevista según los objetivos, el método, el receptor de los cuidados de salud o el canal de comunicación. Según el método de entrevistar, tenemos la entrevista libre, con libertad total del entrevistado para escoger los contenidos a explicar; la dirigida o estructurada, en la que los contenidos están totalmente predeterminados por el sanitario; y la semidirigida o semiestructurada, cuyos contenidos están parcialmente determinados por el sanitario, con espacio libre para la narración del paciente. Es un modelo de EC sencillo y práctico para nuestra realidad diaria y aporta técnicas útiles para llevar a cabo una comunicación eficaz con el paciente y su familia ${ }^{1,2,10}$.

Los pediatras siempre hemos de tener presente la perspectiva del desarrollo porque la capacidad de comunicación de los niños y su comprensión de las enfermedades cambian con la edad. También tiene relevancia la persona que acude como acompañante del paciente de forma habitual (padres, abuelos, cuidadores múltiples, canguros, vecinas, o los "niños de la llave", que se hacen cargo de sus hermanos menores, etc. $)^{3,7,15,16}$.

\section{Entrevista semiestructurada}

La EC semiestructurada (ECS) estructura y ordena, sin rigidez, lo que hacemos todos los días, e incorpora a nuestro quehacer técnicas que nos facilitan el trabajo. A continuación nos vamos a situar en el trabajo diario de un pediatra asistencial, que se enfrenta a un número variable de $\mathrm{EC}$, con diferentes motivos y demandas de salud, también procedentes de pacientes distintos, que nos pueden producir impactos emocionalmente negativos, y todo ello en un tiempo escaso la mayor parte de las veces.

Proponemos un método de trabajo, llamado ECS, que tiene como fin facilitar nuestra practica clínica diaria. Consiste en una secuencia de tareas y habilidades, verbales y no verbales, que siguen un orden lógico y nos permiten abordar satisfactoriamente la mayoría de nuestras consultas. 
Vamos a dividir la EC en cuatro etapas, en las que logramos los fines de toda entrevista ${ }^{1,2,4,16,18,19}$ :

- Antes de empezar la entrevista.

- Parte exploratoria, en la que intentamos averiguar el motivo o motivos de consulta. ¿Recibimos cordialmente?, ¿estamos mirando al ordenador, que se ha colgado?, ¿nos resulta fácil delimitar la demanda y ordenar la consulta? Queremos conseguir conocer la información preelaborada mediante técnicas de apoyo narrativo, que crean un clima de confianza y favorecen la elaboración de ideas o emociones, la búsqueda de datos específicos necesarios para tomar decisiones y el acompañamiento de la exploración física. ¿Nos dirigimos al niño?, ¿sabemos escuchar?, ¿pedimos permiso?, ¿explicamos lo que vamos a hacer?, ¿y el respeto a la intimidad?

- Parte resolutiva, en la que informamos al paciente y sus padres y acordamos un plan de actuación, o intentamos educar en nuevos hábitos, reconvirtiendo si fuera preciso creencias incorrectas, o bien negociando la individualización de las medidas preventivas, terapéuticas o diagnósticas propuestas. Pero infor- mar no es lo mismo que educar. La educación es un proceso en el que tratamos de incorporar o modificar conductas del niño o su familia, en el contexto de una relación humana en la que la imitación, la identificación y la persuasión juegan un papel más importante que la simple información en el logro de cambios en el estilo de vida. ¿Qué características ha de tener la información emitida?, ¿de que técnicas disponemos? Obtenemos la información de los niños pero, ¿a quien informamos nosotros? Implicar al niño facilita que se responsabilice de su salud y de sus hábitos.

- Cierre de la entrevista con una despedida cordial y toma de precauciones. "Consulte de nuevo si no cede la fiebre en tres días"...

\section{Algunas cuestiones demostradas}

- La relación asistencial tiene dos componentes siempre presentes, el humano y el técnico ${ }^{3,8,10-12}$.

- Las habilidades de comunicación son fruto del aprendizaje profesional, más que una capacidad inna$\operatorname{ta}^{1,2,4,9,12}$

- La EC es el método de trabajo fundamental de la atención médica en cualquier especialidad asistencial; 
sentirse poco preparado en comunicación se relaciona con una mayor prevalencia de síndrome de burnout ${ }^{6,7,13,15,20}$.

- Las relaciones profesionales con los pacientes y las familias basadas en una participación activa en la toma de decisiones generan una mayor satisfacción y mejores resultados clínicos ${ }^{4,5,20}$.

- Una relación clínica de calidad aumenta la eficacia en la detección y resolución de problemas clínicos, la satisfacción con la atención recibida, y la adherencia al tratamiento ${ }^{4,8,14,19}$.

- Las habilidades de comunicación ayudan a establecer una relación clínica gratificante para el pediatra, sus pacientes y los padres $11,16,17,20$.

\section{Algunas cuestiones para debatir}

- Las habilidades de comunicación ayudan a contener la demanda asistencial absurda ante cuadros banales.
- Los pacientes y sus padres son/no son nuestros amigos o invitados.

- Puede haber diferentes estilos de entender la consulta y los pacientes, y no tenemos por qué estar todos de acuerdo.

- Es bueno mantener los roles clásicos tales como ser madre, padre, hijo, pediatra...

- La relación clínica como patrimonio de la humanidad.

Partimos del hecho de que todos tenemos ya una forma de entrevistar. Los años de práctica profesional sedimentan un conjunto de conductas $y$, por ello, esta presentación no puede ser esencialmente teórica.

Pretendemos ofrecer al participante un acercamiento a nuevas formas de actuar en el contexto de la EC y a que haga aportaciones desde su propia experiencia. Pero, puesto que comunicar es más un acto eminentemente práctico que teórico, podremos ver unos vídeos con algunos de los casos más habituales en Pediatría.

\section{Bibliografía}

1. Borrell F. Entrevista clínica. Manual de estrategias prácticas. Barcelona: Semfyc; 2004.

2. Ruiz Moral R. Relación clínica: guía para aprender, enseñar e investigar. Barcelona: Semfyc; 2004.

3. Blair AW, Steer CR. Developing communication skills in medicine. Your child is brain dead. Postgrad Med J. 1996;72:137-0. 
4. Cegala DJ, McClure L, Marinelli TM, Post DM. The effects of communication skills training on patients'participation during medical interviews. Patient Educ Couns. 2000;41:209-22.

5. DiMatteo MR, Sherboune CD, Hays RD, Ordway L, Kravitz RL, McGlynn EA et al. Physician's characteristics influence patients' adherence to medical treatment: results from the Medical Outcomes Study. Health Psychol. 1993;12(2): 93-102.

6. Fahrenkopf AM, Sectish TC, Barger LK, Sharek PJ, Lewin D, Chiang VW et al. Rates of medication errors among depressed and burn out residents: prospective cohort study. BMJ. 2008;336:488-91.

7. Mendelsohn JS. Iterview Strategies Commonly Used by Pediatricians. Arch Pediatr Adolesc Med. 1999;153:154-7.

8. Whitcomb ME. Communication and professionalism. Patient Educ Couns. 2000;41:137-44.

9. García-Campayo J, Aseguinolaza L, Tazón P. El desarrollo de las actitudes humanistas en medicina. Med Clin (Barc). 1998;111:23-6.

10. Borrel F. La relación médico-paciente en un mundo cambiante. Humanitas. 2007;15:122.
11. Jovell AJ. Medicina basada en la afectividad. Med Clin (Barc). 1999;113:173-5.

12. Spiro $\mathrm{H}$. What is empathy and can it be taught? Ann Inter Med. 1992;116:843-6.

13. Marinker $M$. The medium and the message. Patient Educ Couns. 2000;41:117-25.

14. Borrell F. Entrevista clínica viva. Aten Primaria. 2004;34:3-5.

15. Pantell RH, Stewart TJ, Dias JK, Wells $P$, Ross W. Physician Communication with Children and Parents. Pediatrics. 1982;70:396-402.

16. McDaniel S, Campbell TL, Seaburn DB. Orientación familiar en Atención Primaria. Manual para médicos de familia y otros profesionales de la salud. Barcelona: Springer-Verlag Ibérica; 1998.

17. Wallace JE, Lemaire JB, Ghali WA. Physician wellness: a missing quality indicator. Lancet. 2009;374:1714-21.

18. White J, Levinson W, Roter D. "Oh, by the way..." The Closing Moments of the Medical Visit. J Gen Intern Med. 1994;9:24-8.

19. Hall JA, Visser A. Health communication in the century of the patient. Patient Educ Couns. 2000;41:115-6.

20. Smith R. Why are doctors so unhappy? BMJ. 2001;322:1073-4. 